\title{
Simulations of W7-X magnet system fault scenarios involving short circuits
}

\author{
M. Köppen*, J. Kißlinger, Th. Rummel, Th. Mönnich, F. Schauer, V. Bykov \\ Max-Planck-Institut für Plasmaphysik, Euratom Association, Teilinstitut Greifswald, Wendelsteinstr. 1, \\ 17491 Greifswald, Germany \\ * Corresponding author: Tel.: +49 383488 1825; fax: +49 3834882439. \\ E-mail address: matthias.koeppen@ipp.mpg.de (M. Köppen)
}

\begin{abstract}
The stellarator Wendelstein 7-X (W7-X) which is presently under construction at the Max-Planck-Institut für Plasmaphysik in Greifswald [1], uses 70 superconducting coils, arranged in 7 groups to create the magnetic confinement for the plasma. A wide variety of tests and investigations are performed in order to ensure the later safe operation of the device. Much attention is also paid to the proper insulation of all the parts. These measures are costly and time consuming but are necessary in order to avoid the severe consequences of faults - especially short circuits - during operation. If a short circuit would happen during an emergency switch-off, the discharge of any shorted inductance would be delayed, and the coupled magnetic flux of the discharging system would induce additional currents into this shorted part. The currents and forces developing in such a case depend not only on the short circuit resistance and the critical current of the superconductor, but also on the shorted inductance itself, its magnetic coupling to other inductances, and its position within the system. The paper describes the influences of these factors and presents simulation results for different fault scenarios involving short circuits across a coil group, a single coil, different double layers, and a single turn. Maximum currents result from a shorted outer double layer, maximum forces from a shorted coil group, depending on its position in the magnet system.
\end{abstract}

Keywords: Stellarator; Superconducting Coils; Simulation; Currents; Forces, Fault

\section{Introduction}

The advanced stellarator Wendelstein 7-X, uses 70 superconducting coils of seven different types to create the necessary magnetic confinement. Ten coils of the same type are always electrically connected in series to form one coil group. Each winding pack of the five non-planar coil types consists of 108 turns that are arranged in six double 
layers, DL. Winding packs, WP, of the two planar coil types comprise three DL, having six turns per layer. Before assembly every coil is tested for short circuits under room temperature and cryogenic conditions. A strong and thoroughly tested insulation is used throughout the system. It should withstand the highest calculated voltages with a sufficient margin. During operation of the W7-X an intelligent handling of detected faults helps to avoid fast discharges whenever possible. Before each magnet operation checkups of the system are planned to test for short circuits to ground and for high voltage weaknesses. All of these measures need time and money, but are nevertheless a necessary investment into the safety of the machine. This paper shows possible consequences, if these measures would be neglected and asymmetries, maximum currents and maximum forces would arise in the system. At first different influences are investigated and resulting currents for worst case scenarios are presented. In a next step these currents are used to calculate the resulting forces that would act on the coils in such situations.

\section{Factors influencing currents, forces and symmetries}

\subsection{Quenches}

The winding packs of the superconducting coils mainly consist of cable in conduit conductors, CICC, which contain the superconducting strands. Inside the CICC supercritical helium is used to keep the strands at a temperature of approx. $4 \mathrm{~K}$, and for thermal stabilisation. If the critical temperature, $T_{c}$, is reached in a part of the conductor, this part looses superconductivity and a quench starts to propagate. During normal operation the temperature across the lateral direction of the WP ranges from $3.4 \mathrm{~K}$ at the inlet side to $4.2 \mathrm{~K}$ at the opposite wall. Heating of the conductor due to changing magnetic fields is neglected in this study. The magnetic flux density which strongly influences $T_{c}$ for a certain current varies from $0 \mathrm{~T}$ somewhere close to the centre of the winding pack to $6 \mathrm{~T}$ at the radial innermost layer of the coil. Due to the non-planar shape of the coils the distribution of B in a cross section also depends on the position along the coil perimeter (see Fig. 1). A quench increases the resistance of the conductor, and since 10 coils of the same type are connected in series, the current - and therefore also the force - for the whole group is reduced. Due to the symmetric distribution of the 
coils across the torus, the currents and forces resulting from a quench are always symmetric. However, the locally increased resistance can lead to an unbalanced voltage distribution.
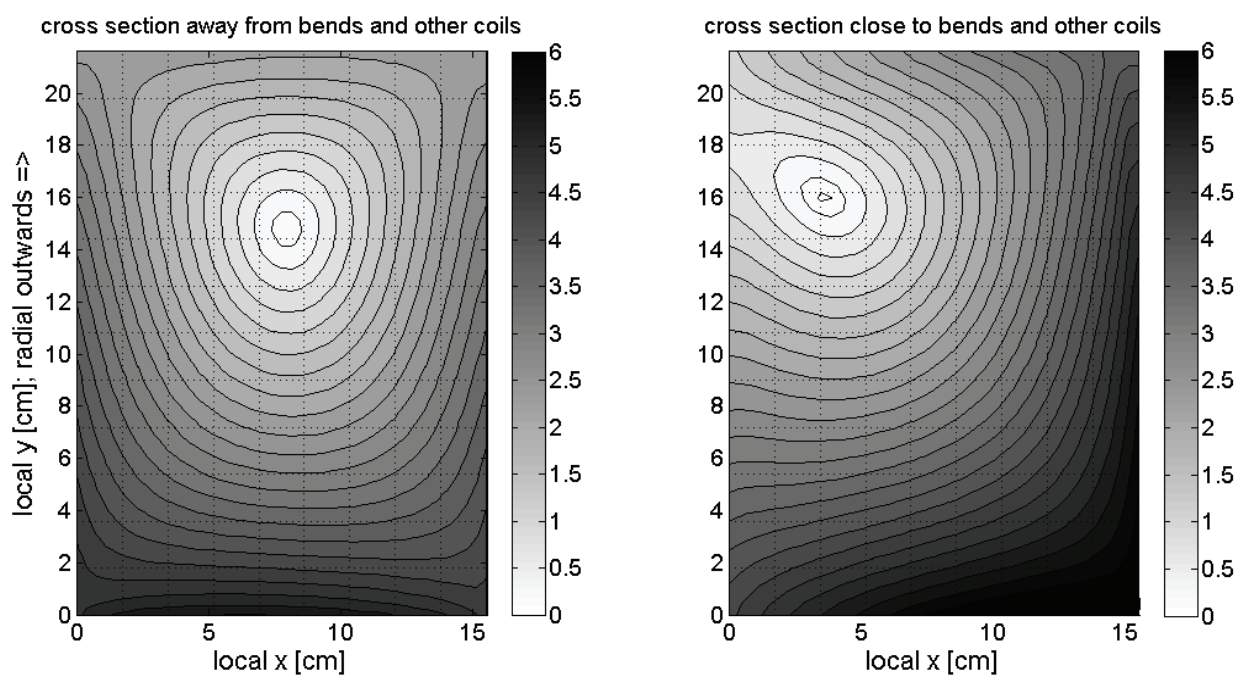

Fig. 1: $|\mathrm{B}|$ at two cross sections of non-planar coil "NPC1" in load case "Low Shear", showing positions of turns (dotted lines)

\subsection{Short Circuits}

In case of an emergency switch off, a shorted inductance discharges only slowly and the quickly changing magnetic field induces additional currents into it. Fig. 2 shows a simplified equivalent circuit diagram for such a case, considering only one group of coils.

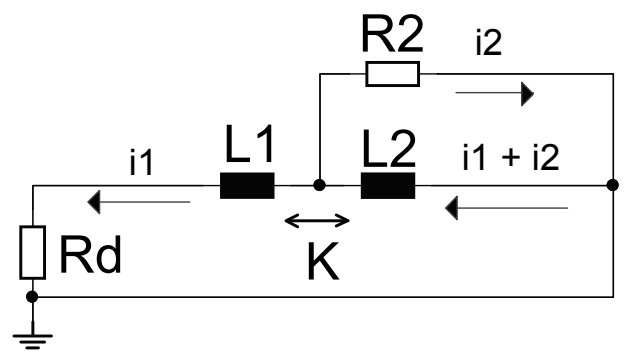

Fig. 2: Simplified equivalent circuit diagram for shorted inductance

While the main inductance of the coil group, L1, is discharged via a dump resistor, $\mathrm{Rd}$, one part of the inductance, L2, is shorted via a short circuit resistance, R2, and it does 
not discharge as quickly as L1. Instead, additional currents, i2, are induced into it due to the magnetic coupling between the two inductances, $\mathrm{K}$, and thus the stellarator symmetry for currents and forces is broken which means that any active short across less than one coil group leads to asymmetries. Solving the appropriate differential equations gives an insight into such a process. Fig. 3 shows how the short circuit maximum current depends on the shorted inductance.

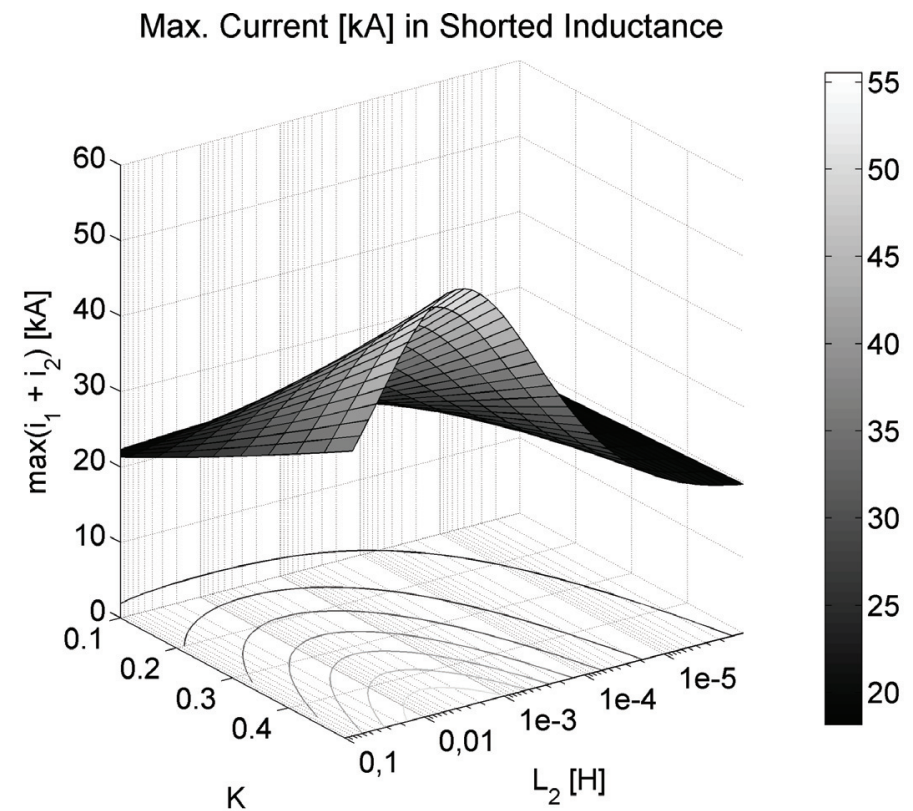

Fig. 3: Maximum currents in shorted inductance for $\mathrm{Rd}=150 \mathrm{~m} \Omega, \mathrm{R} 2=1 \mathrm{~m} \Omega, \mathrm{L} 1=600 \mathrm{mH}$

Comparing this surface with the approximate inductances of W7-X given in Tab. 1, it is easy to find that the maximum current will occur for a shorted inductance in the range of one to two double layers.

Tab. 1: Values for coil group NPC1: approx. shorted inductances, L2, and couplings, K, to rest of group

\begin{tabular}{lcc}
\hline & L2 & $\mathrm{K}$ \\
\hline Coil & $50 \mathrm{mH}$ & 0.08 \\
Inner Double Layer & $1.6 \mathrm{mH}$ & 0.25 \\
Outer Double Layer & $1.9 \mathrm{mH}$ & 0.27 \\
Turn & $8 \mu \mathrm{H}$ & 0.22 \\
\hline
\end{tabular}

Larger inductances lead to a noticeable decrease in the maximum currents, inductances in the range of a single turn show only comparatively weak currents. So the strongest 
currents can be expected for a shorted outer double layer. In the example of Fig. 3 the resistance of the short circuit is kept constant. However, this value also has a major influence on the currents and forces. Smaller resistances cause larger currents and lead to stronger forces.

\subsection{Worst case scenarios}

For this investigation strong forces, which may actually damage the machine, are considered as worst case. Maximum forces develop due to large currents in a strong magnetic field. As seen above, the strongest currents develop for short circuits during a fast discharge of the machine. The maximum current in a shorted inductance mainly depends on: the initial currents, the size and position of the shorted inductance, and the resistance of the short. Here the maximum current of $\mathrm{W} 7-\mathrm{X}, 18.2 \mathrm{kA}$, is used as an initial current, and a small short resistance of $1 \mathrm{~m} \Omega$ is chosen. The results are influenced in two directions depending on the part of the inductance that is shorted. A smaller inductance, e.g. a DL, can lead to stronger currents and thus to stronger forces. However, if only a small portion of a coil has a stronger current, the resulting small volume of increased force density may have only a small influence on the total force acting on the WP. Also, the smaller the shorted inductance, the more turns discharge quickly into the dump resistor. Weak magnetic fields close to the centre of the WP (see Fig. 1) support strong currents but lead only to weak forces. To investigate this trade-off between opposing effects, different scenarios are considered, namely: a short circuit across a whole coil group, a single coil, a single double layer and a single turn of a coil. Strong currents in neighbouring shorted coils lead to maximum forces, and for W7-X the worst case would be a short across two neighbouring non-planar coils of type 1 (NPC1, see Fig. 4) starting with an initial current of $18.2 \mathrm{kA}$. 


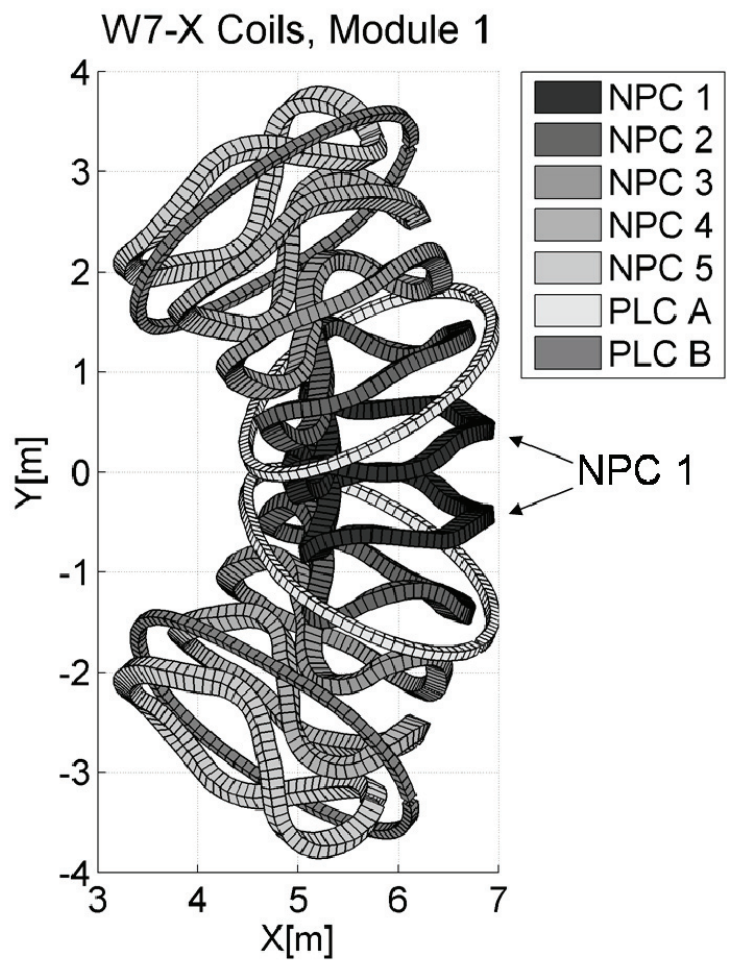

Fig. 4: W7-X: Coils of module 1, two coils of type NPC 1 next to each other

Quenches cause conductor resistivity and thus reduce the currents and therefore also the forces. So for these scenarios the complete absence of a quench would be the worst case. However, above the critical current, quenches are inevitable and have to be taken into account. In these scenarios quenches will occur late and are not able to heat up the conductor too much. A relatively low temperature of $15 \mathrm{~K}$ is assumed for a quenching conductor which leads to a low resistance and thus also to strong currents and forces.

\section{Simulation of Currents}

Using the above worst case parameters, the current development for a fast discharge without quench is simulated (see [1] for details). The resulting currents and magnetic flux densities close to the affected inductances can be compared to the critical currents of the superconductor. Fig. 5 shows these developments of electrical current and magnetic flux density for a shorted outer DL, inner DL, coil, and coil group. 


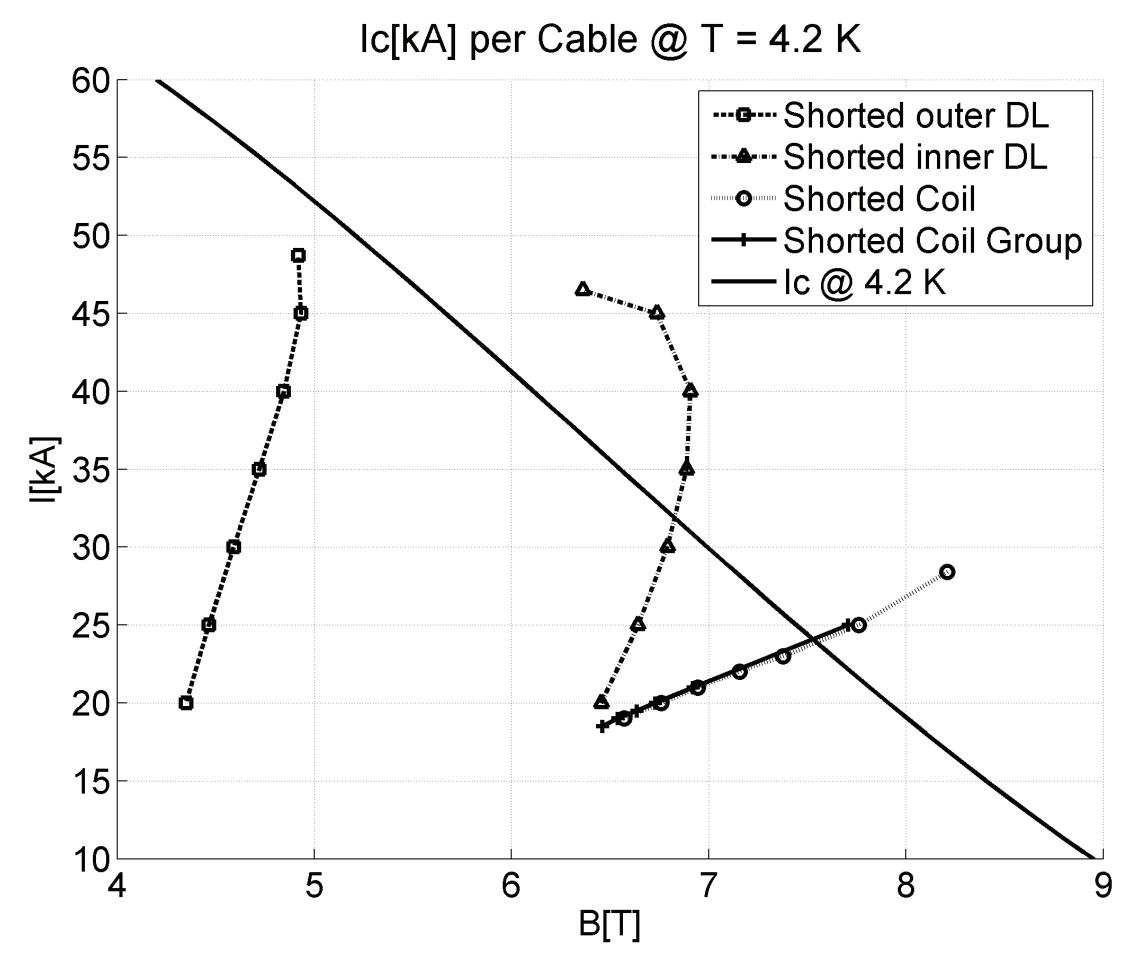

Fig. 5: Development of I and $|\mathrm{B}|$ in shorted inductances and the critical current at $\mathrm{T}=4.2 \mathrm{~K}$

The simulation of a shorted single turn gives an extremely small maximum current of less than $20 \mathrm{kA}$. This is in good agreement with the results described above in 2.2, and this current does not necessarily initiate a quench. As expected, the short circuit across an outer DL produces the strongest current which might not initiate a quench either. Due to the weaker magnetic field in the outer layers (see Fig. 1) strong currents can develop without exceeding the local critical value. Another effect that can be observed in Fig. 5 is the changing correlation between the local current, I, in an inductance and magnetic flux density, B. While B is normally a linear function of I for a coil group, in case of a single shorted coil, the effect of the other discharging coils leads to a slightly less than linear behaviour. If only parts of a coil are shorted, this effect becomes more dominant, i.e. the remaining not shorted turns are partially responsible for the magnetic field close to the shorted inductance. As they discharge, their contribution to the local field decreases and quenching may occur later. Similarly to a shorted outer DL, also a shorted single turn does not lead to currents above the critical one for the superconductor, regardless of its location within the winding pack and the resulting local magnetic field. For all the other cases quenching is inevitable. 
The exact calculation of the propagation and temperature of a quench is complicated and depends on many influences. For this investigation only a very simple model is used: No premature quenching is taken into account and when a quench develops, it affects the whole coil at once. The temperature is kept at a very low level, $15 \mathrm{~K}$. Tab. 2 shows a comparison of the maximum currents in shorted inductances with and without considering quenching.

Tab. 2: Maximum currents and forces for NPC 1 for fast discharge from load case Low Shear

\begin{tabular}{lccc}
\hline & $\begin{array}{c}\mathrm{I}_{\max }(\text { no quench) } \\
{[\mathrm{kA}]}\end{array}$ & $\begin{array}{c}\mathrm{I}_{\max } \text { (quench) } \\
{[\mathrm{kA}]}\end{array}$ & $\begin{array}{c}\text { Maximum total } \\
\text { force on coil [MN] }\end{array}$ \\
\hline $\begin{array}{l}\text { Normal Low Shear } \\
\text { Shorted Turn }\end{array}$ & 18.2 & - & 2.65 \\
$\begin{array}{l}\text { Shorted Inner } \\
\text { Double Layer }\end{array}$ & 19.85 & - & 2.65 \\
Shorted Outer & 46.54 & 39.24 & 2.65 \\
$\begin{array}{l}\text { Double Layer } \\
\text { Shorted Coil }\end{array}$ & 48.54 & - & 2.65 \\
Shorted Coil Group & 28.44 & 25.37 & 3.25 \\
\hline
\end{tabular}

\section{Resulting forces}

For the calculation of the maximum forces, the development of all currents in the system has to be considered. Forces depend on currents and the magnetic field, and the latter may already decrease while some currents still rise. Therefore the maximum forces usually occur earlier than the maximum currents. The currents in all coils are sampled for different time steps during discharge, and the resulting forces are calculated. One example for the changes in force distribution along the coil perimeter is given in Fig. 6. The coil is modelled with 96 segments along the perimeter, and the total forces per coil segment are given for different situations. 


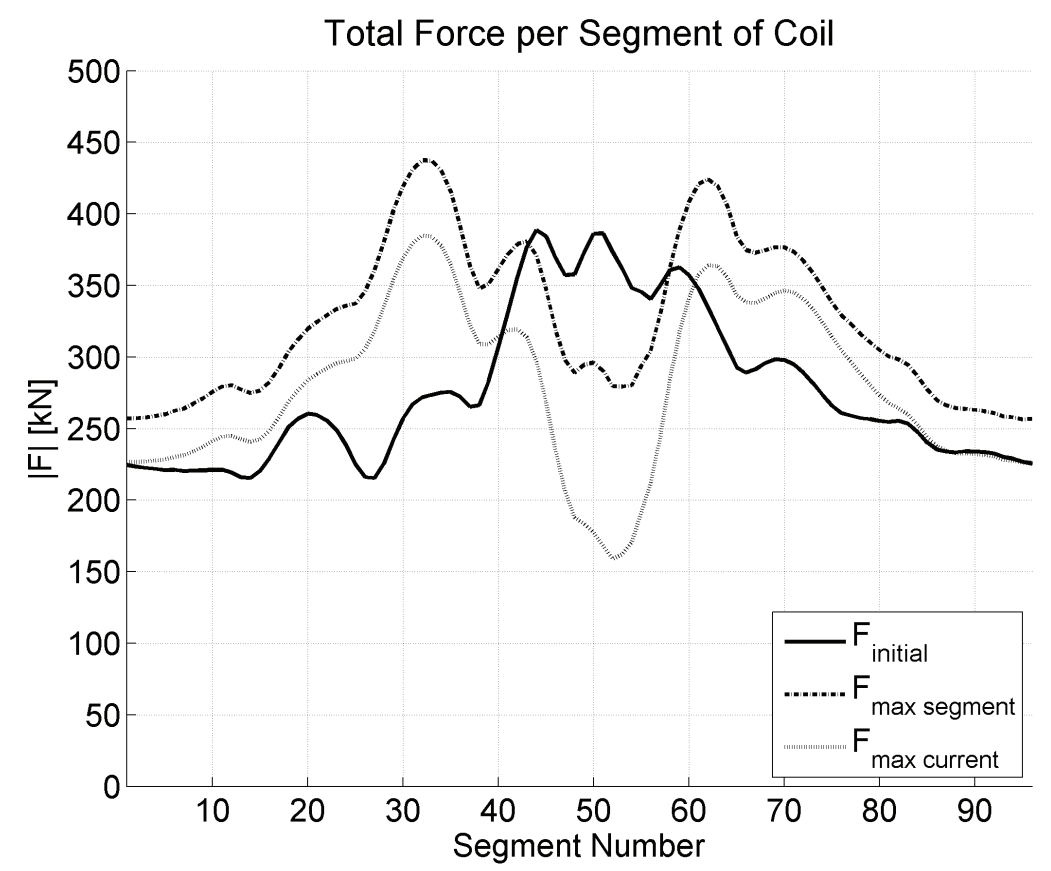

Fig. 6. Forces along perimeter of coil NPC1 for a shorted coil at different times

$F_{\text {initial }}$ shows the total forces per segment during normal operation in Low Shear case. For a shorted coil during fast discharge the maximum total force acting on it, $\mathrm{F}_{\text {max total }}$, is reached after $1.7 \mathrm{~s}$. After $2.9 \mathrm{~s}$ the maximum local force acting on one segment of the coil, $\mathrm{F}_{\max \text { segment, }}$ is reached. The maximum current occurs after $5 \mathrm{~s}$, but at that time the forces, $F_{\max }$ current, already decrease due to the weakening magnetic field. While the local forces acting on the shorted parts increase in all cases, only completely shorted coils increase the total force acting on a coil. In all other cases, the locally increased forces occur at times when the loads in the sound part of the coil already decrease. The local increase is not strong enough to offset the general decline of the forces. Tab. 2 shows the maximum total forces acting on the coil NPC1 for the considered cases. The most dramatic effect occurs for a shorted group of NPC1 coils. Always two coils of this type are positioned next to each other giving five pairs distributed over the torus. Stronger currents in these two neighbouring coils result in strong attractive forces between them (see Fig. 7) which might damage the device. 


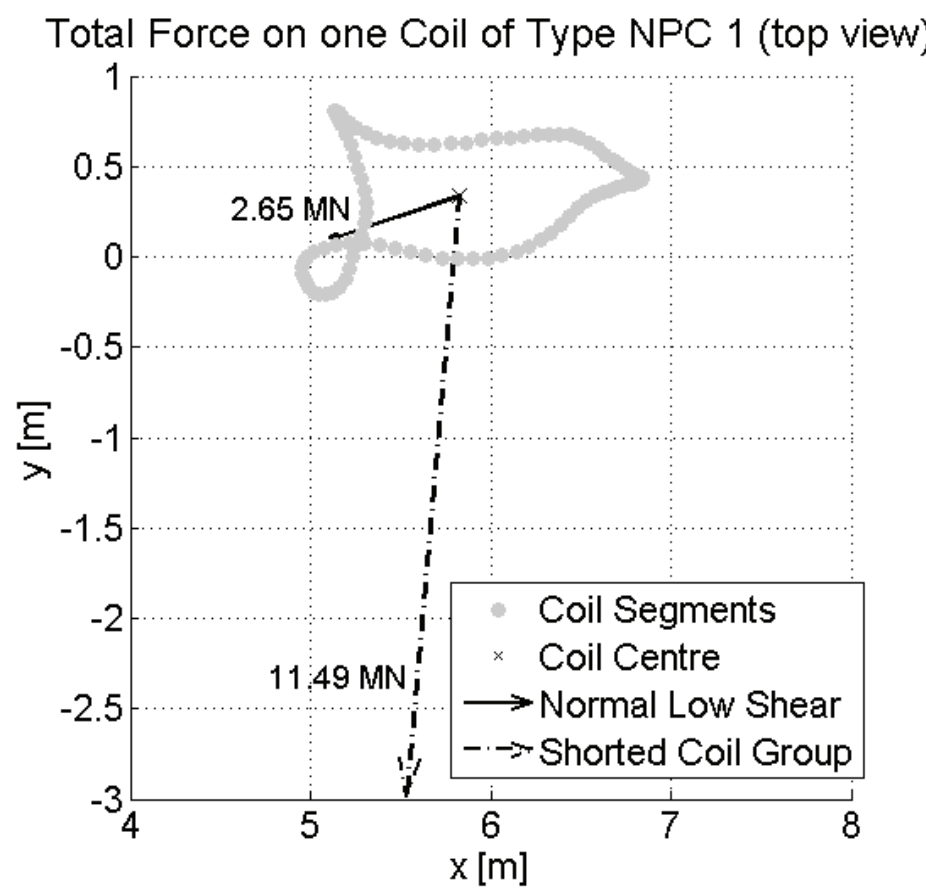

Fig. 7 Increase in total forces on one coil for shorted coil group

\section{Summary and conclusions}

Utmost care is taken to avoid all short circuits during operation of the W7-X. Before assembly every coil is tested for short circuits under room temperature and cryogenic conditions. A strong and thoroughly tested insulation is used throughout the system which should withstand the highest calculated voltages with a large margin. During operation of W7-X, an intelligent handling of detected faults helps to avoid fast discharges whenever possible. Checkups of the system are planned before each magnet operation. These will test for short circuits to ground.

This paper shows the possible consequences of various fault scenarios of the W7-X magnet system. Using circuit simulations, fast discharges of the system are investigated. The correlated current and flux density developments have a strong influence on the occurrence of quenches. Based on these field distributions, simple quench models are then incorporated into the circuit simulations to give realistic current values. While a quench and the subsequent fast discharge lead to a symmetric reduction of currents, short circuits result in strong currents that can be asymmetric. The maximum currents are found for a shorted outer double layer of a coil, while the strongest forces are found for a shorted group of NPC 1 coils. These extreme forces - which would damage the 
device - are mainly due to the small distance between coils of that type, but also to their strong initial current. The aforementioned measures are good and necessary investments into the safety of W7-X in order to avoid the described consequences.

\section{References}

[1] C. Beidler, G. Grieger, F. Herrnegger, E. Harmeyer, J. Kißlinger, W. Lotz, H. Maassberg, P. Merkel, J. Nührenberg, F. Rau, J. Sapper, F. Sardei, R. Scardovelli, A. Schlüter and H. Wobig., Physics and engineering design for Wendelstein 7-X, Fusion Technology, Vol. 17, 148-168, (1990)

[2] M. Köppen, J. Kißlinger, Simulation of Voltage and Current Development in the Wendelstein 7-X Coil System Taking into Account Fault Conditions, Fusion Engineering and Design 82 (2007) 1549-1554 